\title{
The acute effect of a baobab fruit (Adansonia digitata) smoothie on cognitive function in healthy adults
}

\author{
A. Bycroft and S. Coe \\ Functional Food Centre, Oxford Brookes University, Gipsy Lane, Oxford, UK.
}

The baobab fruit is high in both dietary fibre and polyphenols. Results from previous studies show that foods rich in low glycaemic dietary fibre ${ }^{(1)}$ and those that are abundant in polyphenols ${ }^{(2)}$, can improve cognitive function. The aim of the study was to measure the effect of baobab fruit extract on different measures of cognition in healthy adults.

This study was a one-day single blind randomised crossover design ( 3 day washout) conducted on 20 participants (7 men, 13 women; aged 21-40 year; BMI 18-30 kg/m²). Participants were randomised to consume either a test smoothie consisting of $15 \mathrm{~g}$ of baobab extract $(1914 \mu \mathrm{g} / \mathrm{ml}$ polyphenols) or a control smoothie without the addition of baobab $(776 \mu \mathrm{g} / \mathrm{ml}$ polyphenols), on different days in the morning after an overnight fast at Oxford Brooke University. Cognitive tests were administered 90 mins post consumption after a brief familiarisation of the tests. Cognitive measures were based on previous literature into polyphenols, and included executive function utilising the Stockings of Cambridge test ${ }^{(3)}$ and spatial working memory (PsychoPy v1.82) designed by the researchers ${ }^{(4)}$.

Table 1. Mean results for all cognitive measures

\begin{tabular}{|c|c|c|}
\hline Measure & Control & Baobab \\
\hline SOC Problems Solved ( \%) & $94 \cdot 8 \pm 0 \cdot 22$ & $91 \cdot 5 \pm 0 \cdot 28$ \\
\hline SOC Solved in Min Moves ( $\%)$ & $76 \cdot 3 \pm 0 \cdot 42$ & $79 \pm 0.408$ \\
\hline SOC Successful Move (\%) & $94 \pm 0 \cdot 24^{*}$ & $92 \cdot 5 \pm 0 \cdot 26$ \\
\hline SOC Unsuccessful Move ( \%) & $6 \pm 0 \cdot 243^{*}$ & $7 \cdot 5 \pm 0 \cdot 26$ \\
\hline SOC Movement Duration (ms) & $718.59 \pm 868.92$ & $705 \cdot 21 \pm 766 \cdot 83^{*}$ \\
\hline SOC Choice Duration $(\mathrm{ms})$ & $1561 \cdot 37 \pm 2368 \cdot 53$ & $1592 \cdot 62 \pm 2363 \cdot 27$ \\
\hline PsychoPy Corr. Answer Key Resp 1 ( \%) & $39 \pm 0.49$ & $43 \pm 0.49$ \\
\hline PsychoPy Corr. Answer Key Resp 2 (\%) & $45 \pm 0 \cdot 50$ & $42 \pm 0 \cdot 50$ \\
\hline PsychoPy Corr. Answer Overall ( \%) & $84 \pm 0.99$ & $85 \pm 0.99$ \\
\hline PsychoPy Reaction Time Key Resp 1 (s) & $4.52 \pm 1.99$ & $4 \cdot 3 \pm 2 \cdot 62$ \\
\hline PsychoPy Reaction Time Key Resp 2 (s) & $1.07 \pm 1.25$ & $0.77 \pm 1.01$ \\
\hline PsychoPy Reaction Time Overall (s) & $5 \cdot 37 \pm 3 \cdot 24$ & $5.11 \pm 3.63$ \\
\hline
\end{tabular}

Results are means and standard deviations (SD) of all measures after the consumption of control and baobab smoothies in Percentages (\%), Seconds (s) or Milliseconds (ms). Stockings of Cambridge (SOC); Response (Resp). ${ }^{*}=p<0 \cdot 05$ performed significantly better. PsychoPy and SOC test data were analysed using a Wilcoxon - Signed Rank test.

Consumption of the baobab enriched smoothie significantly reduced movement duration $(p<0.05)$ but also increased unsuccessful move type in the Stockings of Cambridge test $(\mathrm{p}<0.05)$. There were no significant differences between testing order. Compared with the taste and nutrient matched control, there were several trends in favour of the baobab smoothie (albeit not significant).

Baobab consumption administered at $15 \mathrm{~g}$ in a smoothie significantly improved reaction time in executive planning tests, but also increased the amount of unsuccessful move types. This research has positive implications for the use of baobab consumption first thing in the morning for improving cognitive function in the hours post prandial.

\section{References}

1. Ingwerson et al. (2007) Appet 49, 240-244.

2. Lamport et al. (2012) Nutr Aging 1, 5-25.

3. File et al. (2005) Menopa 12,193-201.

4. Field et al. (2011) Phys Behav 103, 255-60. 